\title{
JULGAMENTO DE COLINEARIDADE E PERCEPÇÃO DE DISTÂNCIA EXOCÊNTRICA EM CAMPO ABERTO*
}

\author{
Javier Enrique Santillán ${ }^{1}$ \\ Susi Lippi Marques \\ José Aparecido Da Silva
}

Resumo: O propósito foi investigar experimentalmente o desempenho dos observadores numa original tarefa de julgamento de colinearidade em campo aberto (dimensões 20 x 30 m). Participaram do experimento 30 observadores. Os estímulos foram estacas verticais de alturas variáveis, quatro fixos e quatro móveis. A tarefa consistiu em ajustar os dois estímulos móveis (mediante controle à distância) para que ficassem colineares aos dos estímulos fixos nos extremos de cada diagonal. Foi realizada uma análise de variância (ANOVA) fatorial sobre os erros de colinearidade, revelando somente efeito significativo para o fator 'Estímulo Móvel' $\left(F_{(3,473)=} 69,86 p<0,001\right)$ notando-se uma sistemática na distribuição dos erros. As pe-

* Esta pesquisa fez parte do trabalho de doutorado do primeiro autor e teve apoio da Coordenação de Aperfeiçoamento de Pessoal de Nível Superior - CAPES e da Fundação de Amparo à Pesquisa do Estado de São Paulo- FAPESP. Os autores gostariam de agradecer a Odair Batistão e Paulo Aparecido Ambrósio, da Oficina Precisão da PCARP, pela construção dos equipamentos experimentais e a lgor Otto Douchin pelas dicas técnicas. Agradecemos também a Herenilton Paulino Oliveira (Faculdade de Filosofia, Ciências e Letras de Ribeirão Preto da Universidade de São Paulo) pela inestimável colaboração.

1 Atualmente se desempenha no Dpto. de Luminotecnia, Luz y Visión, Faculdad de Ciencias Exactas y Tecnología, Universidad Nacional de Tucumán, Tucumán, Argentina. 
quenas variações na distância exocêntrica percebida (com erros constantes dez vezes menores que os encontrados ao avaliar as distâncias egocêntricas) e a relação dos erros cometidos com as distâncias egocêntricas indicariam que o marco de referência eliciado por esta tarefa de colinearidade, embora possa estar baseado nos indícios exocêntricos, é escalado por um fator egocêntrico.

Palavras-chave: Percepção visual. Percepção espacial. Percepção de distância. Percepção de colinearidade. Psicofísica.

\section{Introdução}

Os estudos da percepção das propriedades geométricas do espaço e dos objetos nele posicionados mostram que, ainda em condições plenas de indícios, os observadores normalmente têm dificuldades para julgar adequadamente as distâncias dos objetos. Alguns trabalhos (Gilinsky, 1951; Fukusima, Loomis, \& Da Silva, 1997; Sedgwick 1986), mostram que os julgamentos de distância egocêntrica (distância de um observador a um objeto) são mais acurados que as respectivas estimativas de distância exocêntrica (distância entre dois objetos, ou partes de um objeto), sugerindo uma dissociação entre os aspectos considerados ao julgar a posição, a separação e a orientação de dois objetos. Contudo, outras investigações apresentam evidência de que os indícios de base exocêntrica podem prover, de forma independente, informação mais acurada que os indícios egocêntricos (Aznar-Casanova, Matsushima, Da Silva, \& RibeiroFilho, 2008). Existem também posturas como as de Loomis, Philbeck e Zahorik (2002) e Foley, Ribeiro-Filho e Da Silva (2004), que tentam conciliar estes resultados.

\section{Percepção de distância e orientação exocêntrica em cam- po aberto}

Pesquisas iniciais usando cenas naturais focaram-se em descrever a percepção de distância egocêntrica (por exemplo, Gilinsky (1951), Gogel (1978), Da Silva (1985)), onde o observador tinha que julgar a distância entre ele e um único alvo que se encontrava dentro de uma configuração. $\mathrm{O}$ interesse no estudo da percepção das distâncias interobjetais na cena e das orientações exocêntricas dos estímulos parece ter sido iniciada em meados dos anos 80 com os trabalhos de Haber (1985), Wagner (1985) e Toye (1986).

As características inovadoras do trabalho de Haber (1985) referemse a uma investigação naturalística, empregando vários objetos (estímu- 
los) apresentados simultaneamente e controlando as distâncias físicas entre eles. Segundo o autor, a descrição da configuração de estímulos foi observada a partir da superfície do terreno e localizadas em termos de coordenadas Euclidianas num espaço tridimensional. Por outro lado, Wagner (1985) indaga outra possibilidade empregando uma distribuição pentagonal de estímulos, onde os sujeitos efetuavam tarefas de estimação de magnitude, igualação e mapeamento. Neste caso, o objetivo foi avaliar as possíveis distorções do espaço visual. Encontrou, então, que a compressão da distância se incrementa a medida que o ângulo da distância observada passa de horizontala radial ${ }^{2}$, com uma compressão de até $50 \%$.Tanto Haber como Wagner tentaram construir uma teoria sobre a forma do espaço percebido partindo da análise das relações entre as distâncias e orientações exocêntricas percebidas.

Toye (1986) realizou sua pesquisa empregando estacas distribuídas de maneira pseudoaleatória. Seus resultados mostraram que os observadores possuem uma representação acurada da cena visual, a qual é independente do método usado nos julgamentos das distâncias, guardando as estimativas uma similaridade estatística com a cena real. Outro achado importante foi que a forma da cena visual percebida muda de acordo com a posição de observação com respeito à configuração, tornando relevante este parâmetro ao definir o layout experimental.

Baseados nestes trabalhos prévios, Levin e Haber (1993) investigaram a percepção de distâncias exocêntricas considerando especificamente os efeitos da aproximação e afastamento da linha de visão do observador. Mas os autores posicionaram as estacas estímulos seguindo um critério específico para conseguir controle sob o ângulo visual e o alinhamento dos segmentos com respeito à linha de visão. Na mesma época, um layout similar foi empregado em nosso laboratório com o objetivo de investigar a acurácia e estabilidade dos julgamentos verbais para estimar distâncias egocêntricas e exocêntricas em configurações espaciais no campo aberto (Ribeiro-Filho, 1993), e cujos dados foram modelados a posteriori no trabalho de Foley et al.(2004). Além de ter sido encontrada uma relação lineal entre as distâncias físicas e as percebidas, Levin e Haber (1993) notaram que a distância exocêntrica apresenta uma distorção que depende do ângulo entre os objetos. Esta distorção produz uma superestimação das distâncias perpendiculares à linha de visão, mas tem pouco efeito nas distâncias paralelas à linha de visão. Ribeiro-Filho (1993) reporta resultados similares, com os julgamentos de distância exocêntrica horizontal tendendo à expansão enquanto os julgamentos de distância exocêntrica radial tendem à compressão.

2 Uma distância ou direção é definida como 'radial' quando está dentro dos 30 o da linha de visão. É definida como 'horizontal' quando se situa dentro dos 300 da perpendicular à linha entre o observador e 0 ponto médio da configuração (Haber, 1985). 


\section{Apontamento exocêntrico e colinearidade}

A diferença das tarefas empregadas nos julgamentos de distância exocêntrica - que tradicionalmente usam configurações com vários estímulos-, os estudos empregando tarefas de apontamento exocêntricose caracterizaram desde o principio por utilizar configurações espaciais baseadas somente num par de estímulos, compostos por um apontador e um alvo.

O apontamento exocêntrico bem pode ser pensado como um paradigma (Koenderink \& van Doorn, 1998), pois agrupa na sua definição vários tipos de tarefas, inclusive as de paralelismo ${ }^{3}$ e de colinearidade ${ }^{4}$, as quais podem ser consideradas como casos particulares. $O$ apontamento pode ser executado orientando um segmento a um ponto, um segmento a um outro segmento de reta, dois segmentos a um ponto. No caso do paralelismo, ambos segmentos devem estar orientados na mesma direção, mas pertencer a linhas diferentes. Na tarefa de colinearidade os três (ou mais) pontos, como os segmentos por eles definidos, devem determinar uma reta.

Uma revisão na literatura permite observar que o emprego de tarefas de apontamento para realizar julgamentos de distância exocêntrica encontra-se em estado incipiente, sendo, portanto, os estudos realizados concebidos como inovadores. Destacam-se os trabalhos pioneiros do grupo de investigação liderado pelo professor Jan Koenderink na Universidade de Utrecht (nos Países Baixos).

\section{Tarefas de colinearidade em campo aberto}

Um trabalho que pode ser considerado como precursor no emprego de tarefas de apontamento exocêntrico no campo aberto foi apresentado em 1997 num congresso sobre "Visão e Ação" na Universidade de New York por Koenderink e van Doorn (1998). O objetivo era estudar os aspectos projetivos da estrutura do espaço visual de forma independente de qualquer propriedade métrica. Neste experimento o alvo e o ponteiro foram posicionados em dois dos vértices de um triângulo equilátero com o observador posicionado no baricentro ou centro geométrico da configuração. As distâncias variavam entre 1,5 e 24 metros. O ângulo visual subtendido entre o alvo e o ponteiro foi de $120^{\circ}$, o que forçava o observador a mover a cabeça para conseguir ver ambos claramente. Os mesma direção e que por mais que se prolonguem não podem se encontrar.

4 Colinearidade é a propriedade que indica que em um conjunto de três ou mais pontos, eles estão posicionados de tal forma que se pode traçar uma reta que contenha todos eles. 
resultados mostram um comportamento simultâneo de compressão e expansão, variando em função das distâncias exocêntricas consideradas. Os triângulos pequenos aparecem "inflados", excedendo a soma de seus ângulos os $180^{\circ}$ axiomatizados pela geometria euclidiana. Contrariamente, os triângulos grandes aparecem "desinflados", pelo que a adição de seus ângulos seria inferior aos $180^{\circ}$ euclidianos. Segundo os autores, o excesso na estimativa angular indicaria uma curvatura elíptica e o"déficit angular" indicaria uma curvatura hiperbólica. Este resultado seria coerente com a proposta de Luneburg (1947) de um espaço visual de curvatura não constante e cuja percepção fora caracterizada pela função sugerida por Gilinsky (1951).

Uma variante interessante de tarefa de colinearidade foi a proposta por Kelly, Loomis e Beall (2004), onde a tarefa do sujeito foi observar dois alvos (com separações de 45,90 e $135^{\circ}$ e distâncias egocêntricas fixas de $5,10,15$ e $20 \mathrm{~m}$ ) e sinalizar um terceiro ponto na distância que fosse colinear a eles. Para isso, o observador tinha que imaginar a linha conectando os alvos e depois extrapolar dita linha imaginada até uma cerca perimetral que delimitava o campo. As respostas eram marcadas num cilindro com uma foto panorâmica de dita cerca. Estes autores encontraram um erro médio de só $7,43^{\circ}$, o que, segundo eles, indicaria a boa precisão dos julgamentos, resultado que foi surpreendente devido aos grandes ângulos que o observador devia percorrer com a visão desde um extremo a outro da reta de colinearidade. A similaridade nos padrões de erros com aqueles observados nos estudos de Cuijpers, Kappers e Koenderink (2000a) no laboratório leva a supor que as tarefas empregadas baseiam-se no mesmo tipo de julgamento exocêntrico.

Mais recentemente, Koenderink, van Doorn, Kappers, Doumen e Todd (2008) empregaram no campo aberto uma variante da tarefa de apontamento que seu grupo de trabalho usara com sucesso no espaço reduzido do laboratório (Cuijpers et al.2000a, 2002). O objetivo foi investigar a existência de um arco"pregeodésico" 5 - único e reto - conectando cada par de pontos do espaço, representados pelo ponteiro e o alvo e a respectiva inversão dos mesmos. Neste caso o posicionamento dos estímulos seguiu uma configuração geométrica triangular, com o ponteiro, alvo e observador em cada um dos vértices. As distâncias foram de 1,2,4, 8,16 e 32 metros fixando o ângulo interior no'egocentro'em $60^{\circ}$.O tamanho angular dos estímulos foi mantido constante em $1,8^{\circ}$, modificando $o$ tamanho físico dos estímulos. Contudo, os autores notaram que mesmo

5 Nas matemáticas, a noção de "geodésico" é uma generalização da noção de 'linha reta' num espaço curvo. 0 arco "pregeodésico" é uma curva definida de forma tal que todas suas tangentes são paralelas no sentido geométrico, mas que se diferencia do geodésico por não implicar uma propriedade métrica e sim projetiva. Assim um arco pregeodésico no espaço visual apareceria como uma linha reta, mas no espaço físico seria mapeado como curvo (embora possa apresentar pontos de inflexão). 
assim os observadores percebiam a diferença real de tamanho entre os estímulos próximos e distantes. Os resultados mostraram marcadas diferenças individuais, com variações muito maiores quando o ponteiro estava próximo do sujeito que quando estava longe. Embora não seja possível extrapolar os resultados para os dados obtidos nos experimentos no laboratório (Cuijpers et al., 2000a, 2002) devido às diferenças contextuais e metodológicas, é interessante notar que em geral a informação obtida parece mostrar a incapacidade dos observadores para estabelecer arcos simples no espaço visual.

Devido às implicações, torna-se importante aprofundar o estudo sobre nossa percepção visual ante tarefas que exijam seguir uma linha reta no espaço físico, tais como as de apontamento exocêntrico ou colinearidade, especialmente num ambiente naturalístico e pleno de indícios como é o campo aberto.

O propósito do presente estudo foi investigar experimentalmente o desempenho dos observadores numa tarefa de colinearidade em campo aberto, em condições de indícios múltiplos e com ângulos visuais amplos.

Neste experimento, em vez do posicionamento acostumado nas pesquisas de distância exocêntrica, que normalmente é radial ou horizontal com respeito à linha de visão do observador (paralelo ou perpendicular aos eixos cartesianos), o arranjo dos estímulos foi estabelecido seguindo aproximadamente as duas diagonais no espaço empregado, abarcando um ângulo amplo no campo visual. Embora a geometria básica seja parecida à empregada por Koenderink et al. (2008), pode-se dizer que neste caso os triângulos definidos pelos estímulos se encontram de forma simultânea no espaço físico e numa reflexão não simétrica a partir do ponto central da configuração.

\section{Metodologia}

\section{Participantes}

Participaram do experimento 30 observadores (metade do sexo masculino, metade do sexo feminino), alunos de cursos de graduação e pós-graduação da FFCLRP.O intervalo de idades foi de 20 a 34 anos, com uma média para as mulheres de 25,4 anos e de 27,4 anos para os homens. Todos com visão normal ou corrigida, ingênuos quanto à natureza do experimento, sendo sua colaboração voluntária. A proposta de estudo foi submetida e aprovada pelo Comitê de Ética da USP-RP (Protocolo CEPFFCLRP n 224/2005 - 2005.1.1657.59.0) e pelo SINESP do Ministério de Saúde (Protocolo n ${ }^{\circ}$ 0992.0.222.000-05). 


\section{Ambiente experimental}

Campo aberto plano de dimensões $20 \times 30 \mathrm{~m}$, onde foi realizada uma terraplanagem e coberto com uma camada uniforme de areia compactada. A área de aplicação do estudo foi delimitada por uma pequena mureta de tijolos com uma altura aproximada de $15 \mathrm{~cm}$. Em uma das laterais de $20 \mathrm{~m}$ foi construída uma base de concreto de $0,5 \times 1 \mathrm{~m}$ onde ficava o observador e onde uns parafusos (chumbados perto do lado mais interno ao campo) permitiam a correta fixação do suporte de queixo (ver Figura 1). Do local de posicionamento do observador eram visíveis à esquerda e na frente algumas árvores e mato. Já à direita podiam ser avistadas algumas árvores, existindo também uma rua interna transitada ocasionalmente por veículos e pedestres. No período de coleta de dados o clima foi ótimo com condições de sol pleno em quase todas as medições. As sessões de coleta foram realizadas, na sua totalidade, no período da manhã, com o propósito de que alguns indícios (por exemplo, as sombras) ficassem na mesma orientação e mantidos para todos os participantes.

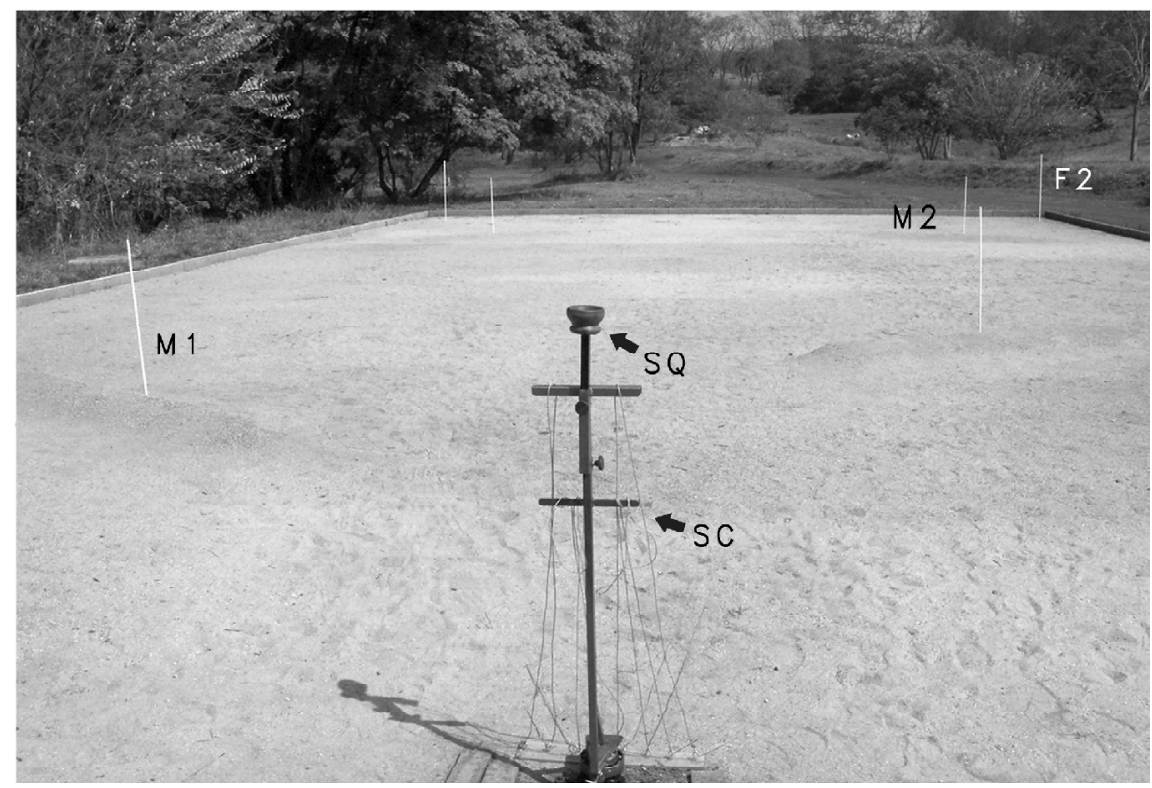

Figura 1: Foto da área experimental desde a posição do observador. Observa-se o suporte de queixo (SQ) e o sistema de controle (SC) usado para ajustar os estímulos móveis (o qual ficava dissimulado na areia). A modo de exemplo aparecem marcados só três dos estímulos da Diagonal 1 (M1, F2 e M2). 


\section{Equipamentos e materiais}

De forma similar a Foley et al. (2004), os estímulos foram estacas de alturas variáveis (desde 1,10 a 1,70 m com uma média de 1,32 m) e diâmetro de 1,25 cm, todas pintadas na cor branca (Figura 1). Os estímulos de posição ajustável foram montados sobre uma base móvel encarrilhada em trilhos invisíveis ao observador do seu ponto de observação. Os trilhos foram posicionados perpendicularmente à extensão entre os pontos terminais que eram fixos ( $F 1$ e F2, F3 e F4), como pode ser observado na Figura 2. Os trilhos foram posicionados descentralizados em relação à linha de colinearidade, de forma que ela não passasse pelo centro de equilíbrio do trilho, evitando assim que esta proporção pudesse ser empregada como referência na hora do observador realizar os ajustes. A distribuição dos estímulos no campo buscou manter certa simetria a partir do ponto central da configuração (localizado a $12 \mathrm{~m}$ na frente do sujeito), mas as distâncias interestímulos e o posicionamento foram ligeiramente diferentes em cada quadrante para evitar que dita regularidade fosse usada como referência por parte do observador.

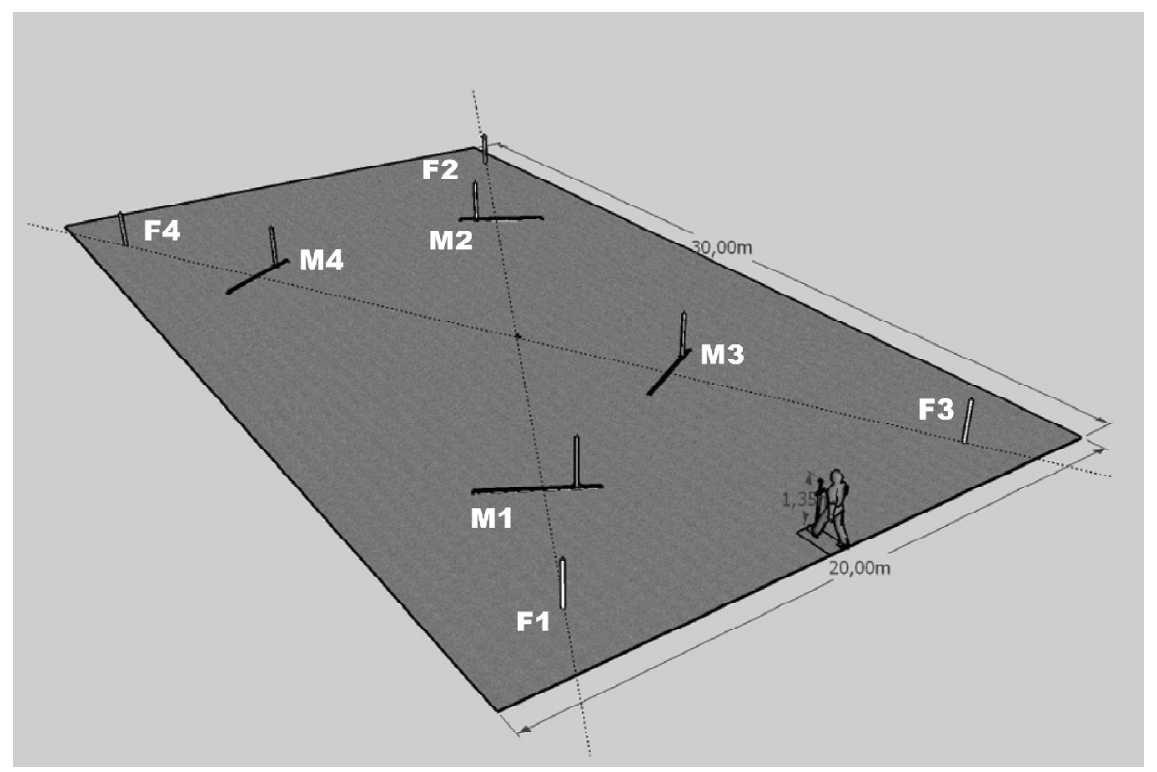

Figura 2: Esquema a escala da distribuição dos estímulos no campo aberto em perspectiva aérea ( $M=$ móvel, $F=$ fixo). Indicam-se as linhas de colinearidade para cada serie de estímulos e a posição do sujeito com o suporte de queixo.

Os estímulos móveis (M1, M2, M3 e M4) foram ajustados através de um sistema de controle à distância, mediante fios dissimulados no solo que permitiam mover os polos verticais de um extremo ao outro do tri- 
Iho. Como o erro na fixação do trilho na sua posição foi de $0,5 \mathrm{~cm}$, no levantamento das medições se considerou este valor como a precisão do sistema. As Tabelas 1 e 2 sumariam as distâncias entre os estímulos de cada diagonal e entre cada um deles e o ponto de observação $O$, determinado pelo centro do suporte de queixo, ou distância egocêntrica física. Os valores estimados para os ângulos visuais entre F1 e F2, e entre F3 e F4, foram de $\grave{e ̀}_{F 1 F 2}=94^{\circ}$ e è ${ }_{F 3 F 4}=101^{\circ}$, respectivamente. Estes valores foram confirmados com medições no campo, com um erro aproximado de $\pm 2^{\circ}$ devido ao sistema empregado.

Tabela 1. Distâncias entre os estímulos da reta de colinearidade que passaria por F1, M1, M2 e F2. Se indica também a distância de cada estímulo ao ponto O (distância egocêntrica). 1 Distâncias entre os estímulos da reta de colinearidade que passaria por F1, M1, M2 e F2. Se indica também a distância de cada estímulo ao ponto $\mathrm{O}$ (distância egocêntrica).

\begin{tabular}{cccccc}
\hline & F1 & M1 & M2 & F2 & O \\
\hline F1 & - & 4,25 & 22,80 & 29,25 & 6,00 \\
M1 & 4,25 & - & 18,55 & 25,05 & 6,10 \\
M2 & 22,80 & 18,55 & - & 6,50 & 21,56 \\
F2 & 29,25 & 25,05 & 6,50 & - & 28,23 \\
\hline
\end{tabular}

Tabela 2. Distâncias entre os estímulos da reta de colinearidade que passaria por F3, M3, M4 e F4. Se indica também a distância de cada estímulo ao ponto O (distância egocêntrica). 2 Distâncias entre os estímulos da reta de colinearidade que passaria por F3, M3, M4 e F4. Se indica também a distância de cada estímulo ao ponto $\mathrm{O}$ (distância egocêntrica).

\begin{tabular}{cccccc}
\hline & F3 & M3 & M4 & F4 & O \\
\hline F3 & - & 8,00 & 23,25 & 29,25 & 6,00 \\
M3 & 8,00 & - & 15,25 & 21,25 & 8,05 \\
M4 & 23,25 & 15,25 & - & 6,00 & 21,71 \\
F4 & 29,25 & 21,25 & 6,00 & - & 27,54 \\
\hline
\end{tabular}

Pelo fato de os alvos serem facilmente identificáveis na sua localização espacial, para simplificar o aspecto visual da configuração de estímulos não foram utilizadas placas de identificação, evitando-se também introduzir no campo objetos que poderiam servir como referência de tamanho.

Um suporte de queixo fixado a uma altura de 1,35 m foi empregado para restringir os movimentos do observador, especialmente no sentido vertical e translacional de paralaxe, mas podia ser girada a cabeça 
com liberdade permitindo-se o movimento de vaivém (paralaxe rotacional) necessário para abarcar todos os estímulos. Assim sendo, a totalidade dos sujeitos observou a configuração de estímulos do mesmo ângulo de visão.

\section{Planejamento experimental}

Foi empregado um planejamento fatorial de um fator intra-sujeitos, duas direções de ajustamento (partindo de Dentro ou partindo de Fora ${ }^{6}$ ) e duas séries de estimativas, considerando como variável dependente o erro no ajuste de colinearidade. $\mathrm{Na}$ análise dos dados foram consideradas também como variáveis de interesse a posição espacial dos estímulos móveis (devido às diferenças próprias do campo aberto e da configuração de estímulos), e o sexo dos observadores.

\section{Procedimento}

Após a aceitação para participar, o observador era conduzido até o local de pesquisa. Todos os observadores assinaram um Termo de Consentimento Livre e Esclarecido. Em seguida, eram acomodados numa posição à parte do campo experimental onde eram lidas as instruções, do tipo objetiva, que solicitavam que os observadores ajustassem a posição dos estímulos móveis $\mathrm{M} 1$ e $\mathrm{M} 2$ de modo que ficassem colineares à extensão compreendida entre F1 e F2, seguind o o mesmo esquema para os estímulos M3 e M4 mantendo-os colineares à extensão compreendida entre F3 e F4. As instruções incluíam figuras com esquemas da tarefa a ser realizada e enfatizavam que as posições poderiam ser ajustadas 0 quanto desejassem. Era esclarecida também qualquer dúvida que surgia no momento, e permitida uma breve prática na movimentação dos estímulos móveis.

Durante os ajustamentos o observador ficava em pé e com a cabeça apoiada no suporte de queixo. Os controles dos estímulos ficavam perto das mãos e organizados de acordo com a posição dos mesmos, mas era permitido ao observador acomodá-los de outra forma se ele achasse que isso lhe facilitava seu uso e/ou manuseio.

Iniciado o experimento, o observador virava de costas e o experimentador posicionava adequadamente os estímulos no ponto de início (aleatório) indicado pelo protocolo. Então, o observador começava a tarefa de ajuste dos alvos. Após cada série de ajustes dos quatro estímulos móveis, os observadores viravam de costas durante o período de medi-

6 Entendendo aqui por 'Fora' ou 'Dentro' se o alvo móvel era posicionado para começar os ajustes na metade externa ou interna do trilho, separação definida a partir do ponto 0 onde ele era cruzado pela linha de colinearidade. 
ção das posições dos estímulos ajustáveis, de forma que não pudessem utilizar o corpo do experimentador como um referencial de tamanho e de distância.

Os erros na colinearidade foram medidos em centímetros a partir do ponto 0 de cada trilho, que era onde passava a linha reta que unia todos os estímulos em cada diagonal ( $F 1 F 2$ e $F 3 F 4$ ). O erro era considerado negativo no sentido proximal ao observador e positivo no sentido oposto.

\section{Resultados}

Os dados oriundos dos ajustes de colinearidade foram codificados em termos de desvio em centímetros da linha de colinearidade. Os resultados foram organizados por estímulo móvel, sexo dos observadores, ponto de início da medição e encontram-se sumariados na Tabela 3.

Tabela 3. Erros de colinearidade relativos ao Experimento $(\mathrm{N}=480)$. Erros médios e desvios-padrão, medianas e rango interquartílico, expressados em centímetros. Os resultados estão discriminados por Estímulo Móvel (1 a 4), Sexo do observador ( $M$ ou F) e Ponto de Inicio da medição (Fora ou Dentro)

\begin{tabular}{ccccccc}
\hline E. Móvel & Sexo & Inicio & Média & SD & Mediana & RIQ \\
\hline 1 & $\mathrm{M}$ & $\mathrm{F}$ & $-10,03$ & 35,29 & $-7,75$ & 46,00 \\
1 & $\mathrm{M}$ & $\mathrm{D}$ & $-14,75$ & 38,51 & $-7,50$ & 45,38 \\
1 & $\mathrm{~F}$ & $\mathrm{~F}$ & 2,68 & 37,31 & 6,00 & 51,50 \\
1 & $\mathrm{~F}$ & $\mathrm{D}$ & $-1,45$ & 34,38 & $-3,00$ & 54,38 \\
2 & $\mathrm{M}$ & $\mathrm{F}$ & $-41,72$ & 20,61 & $-42,50$ & 24,13 \\
2 & $\mathrm{M}$ & $\mathrm{D}$ & $-30,85$ & 23,08 & $-32,00$ & 37,00 \\
2 & $\mathrm{~F}$ & $\mathrm{~F}$ & $-54,37$ & 30,95 & $-46,00$ & 45,38 \\
2 & $\mathrm{~F}$ & $\mathrm{D}$ & $-42,70$ & 38,63 & $-44,50$ & 61,75 \\
3 & $\mathrm{M}$ & $\mathrm{F}$ & 16,08 & 35,68 & 20,25 & 47,13 \\
3 & $\mathrm{M}$ & $\mathrm{D}$ & $-1,72$ & 33,94 & 8,50 & 38,38 \\
3 & $\mathrm{~F}$ & $\mathrm{~F}$ & 12,47 & 42,04 & 15,50 & 46,13 \\
3 & $\mathrm{~F}$ & $\mathrm{D}$ & 9,27 & 43,09 & 16,25 & 51,25 \\
4 & $\mathrm{M}$ & $\mathrm{F}$ & $-34,85$ & 25,82 & $-42,00$ & 26,25 \\
4 & $\mathrm{M}$ & $\mathrm{D}$ & $-23,88$ & 22,71 & $-20,75$ & 30,50 \\
4 & $\mathrm{~F}$ & $\mathrm{~F}$ & $-34,71$ & 31,99 & $-41,75$ & 28,13 \\
4 & $\mathrm{~F}$ & $\mathrm{D}$ & $-22,07$ & 30,27 & $-25,50$ & 41,38 \\
\hline Todos os & & & $-16,90$ & 38,09 & $-20,00$ & 56,50 \\
grupos & & & & & & \\
\hline
\end{tabular}

Uma primeira inspeção, considerando o conjunto dos dados, indicaria um erro de colinearidade negativo com Média = -16,91 e Mediana = $-20,00$. Pode ser observado que tanto as médias $(-17,64$ nos homens, - 
16,16 nas mulheres) como as medianas $(-20,00$ nos homens, $-20,75$ nas mulheres) dos erros são negativas para ambos sexos. Consideram-se aqui as medianas como dado relevante devido ao fato que alguns dos observadores realizaram julgamentos aparentemente fora da distribuição normal dos demais e essa medida de tendência central é menos afetada pelos outliers. De forma similar a Koenderink et al. (2008), ao olhar os dados discriminados por observador, encontramos marcas das diferenças individuais.

$\mathrm{Na}$ Figura 3 foram representadas as medianas e os intervalos interquartílicos. O boxplot permitiu identificar os outliers (Indicados com um * na figura), definidos aqui como aqueles valores cuja distância à mediana é $>1,5$ vezes o intervalo interquartílico. Devido ao fato de que estes valores extremamente inusuais afetariam a divisão dos dados e sua remoção não altera a média significativamente - já que a mudança está na casa do erro de medição $(0,5 \mathrm{~cm})$-, foram removidos dos dados antes da análise estatística das diferenças entre os grupos de observações. Como a posição espacial é um dos fatores de maior interesse em nossa pesquisa o desempenho foi descriminando segundo os estímulos móveis. Assim, observa-se que as mulheres têm erro positivo no M1 (embora não seja significativo por ser da mesma ordem de magnitude do erro de medição), no entanto, ambos os sexos apresentam erros positivos no estímulo M3.

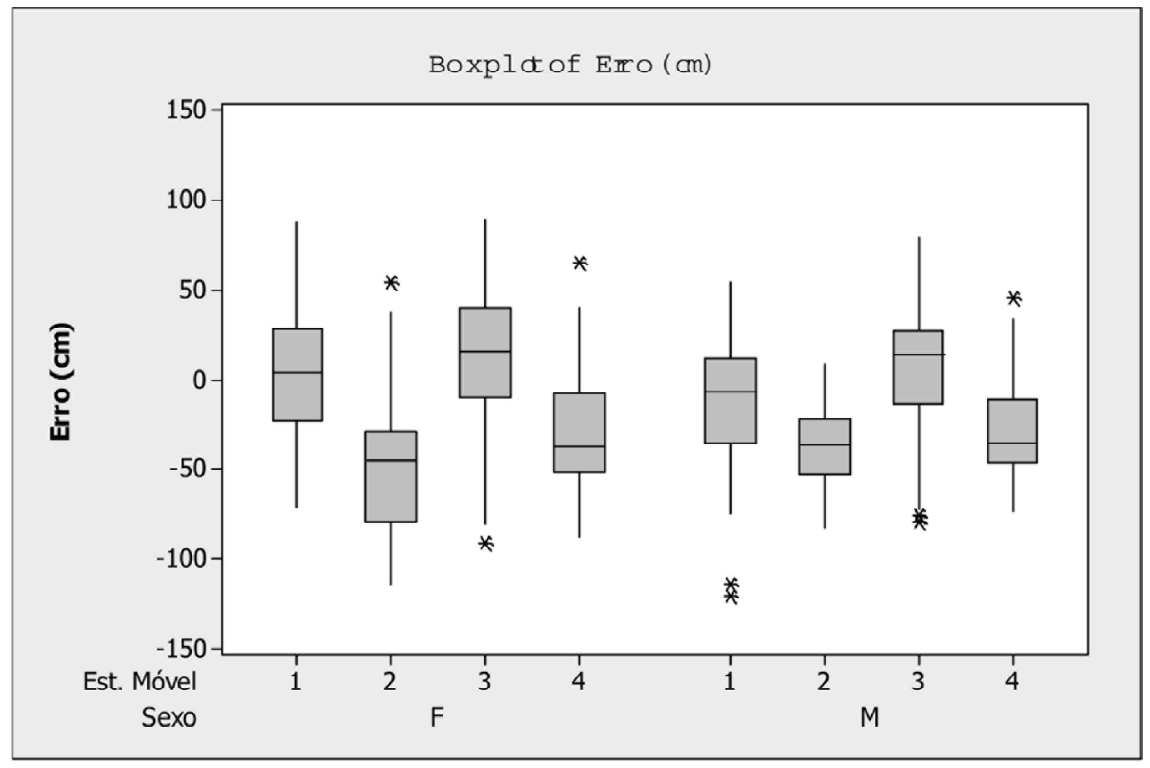

Figura 3: Boxplot dos erros (em centímetros) cometidos no ajuste de colinearidade separados por posição espacial (estímulo móvel) e por sexo do observador. Os outliers são indicados com um '*' 
Segundo pode ser observado, $67 \%$ das observações têm valores negativos, ficando mais da metade dos resultados (55\%) categorizados entre 0 e $-50 \mathrm{~cm}$. A análise de normalidade foi realizada usando o teste de Kolmogorov-Smirnov $(D=0,447, p>0,05)$, o qual indica que a distribuição dos erros segue um padrão similar à curva normal.

Uma vez analisada a normalidade das observações, realizou-se um estudo sobre a igualdade na variância dos erros segundo a posição espacial (estímulo móvel), o sexo do observador e o ponto de início (fora (F) ou dentro (D)) das mesmas empregando o teste de Levene. Em todos os casos não houve diferenças entre as variâncias e o teste revelou homogeneidade em todos os casos com $p>0,05$.

Dando continuidade, foi realizada uma análise de variância (ANOVA) fatorial sobre os erros de colinearidade, considerando os fatores principais Alvo Móvel, Ponto de início da medição (Fora o Dentro), Sexo do observador, Ordem da medida (primeira ou segunda). A análise revelou somente efeito significativo para o fator 'Estímulo Móvel' com $F_{(3,473)=} 69,86, p<0,001$. O estudo das interações entre os efeitos principais denota como significativa a interação entre 'Estímulo Móvel' e o 'Ponto de Início da medição', $F_{(3,448)=} 3,413, p<0,05$.

Nas duas variáveis onde o ANOVA resultou em valores significativos de $F$ foi realizada uma análise pós-hoc. No caso do fator Estímulo Móvel, o teste de Newman-Keuls encontra diferenças significativas $(p<0,05)$ para as quatro posições espaciais; entretanto, para a interação 'Estímulo Móvel*Ponto início medição' a diferença significativa se encontra no estímulo M4.

Na Figura 4, foram traçados os erros em função da distância egocêntrica ao ponto de colinearidade de cada um dos estímulos. Indicamse as relações entre os estímulos, ou seja, sua pertença a cada uma das diagonais do sistema de estímulos. Além das diferenças já apontadas pela ANOVA, nota-se uma sistemática na distribuição dos erros. Para investigar a mesma, foi realizada uma análise empregando regressão linear dos resultados obtidos, separados por sua vinculação espacial. 


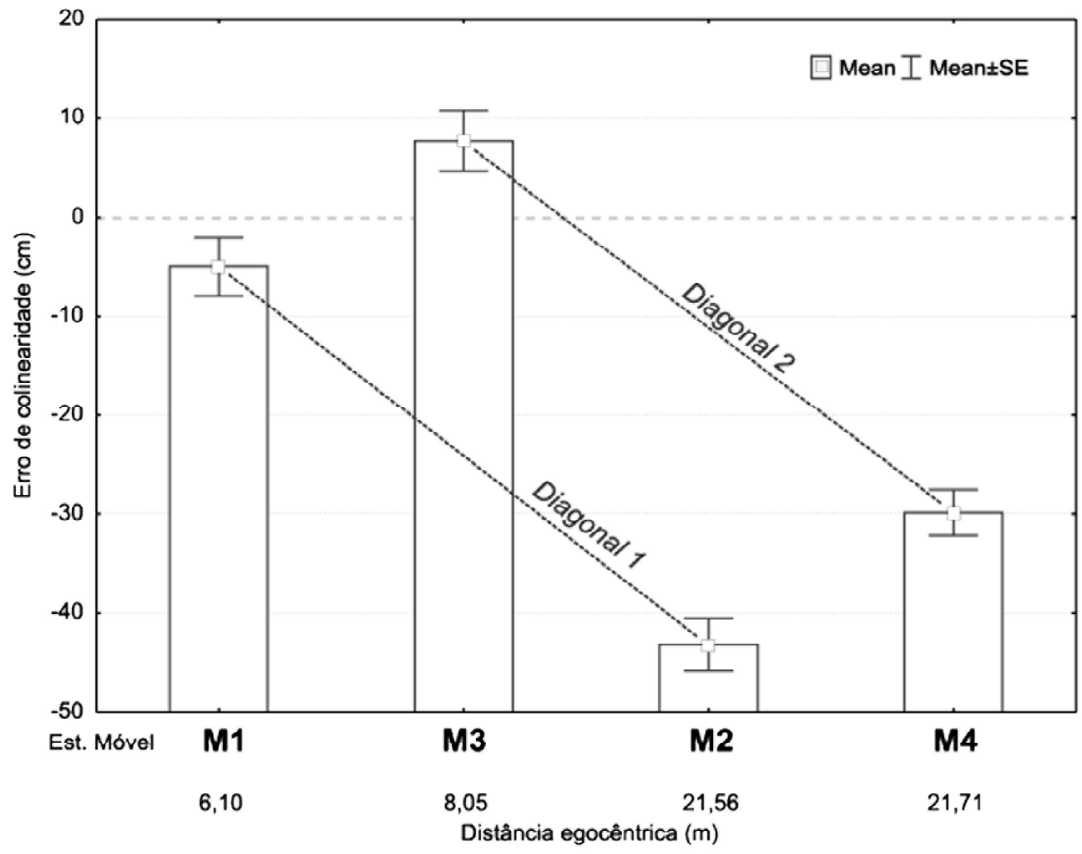

Figura 4: Gráfica da média dos erros de ajuste de colinearidade (em centímetros) separados por posição espacial (estímulo móvel) e distância ao observador do ponto de colinearidade física. Se indicam as Diagonais do sistema de estímulos. As barras verticais indicam o erro padrão da média (SE).

A reta de regressão produz um ajuste dos dados numa função linear do tipo $y=a x+b$, onde o parâmetro $a$, ou inclinação, se refere à precisão do ajuste entre as respostas dos dois estímulos e o parâmetro $b$, ou interseção, indicaria um erro constante, associado ao ajuste de colinearidade e independente da distância física do alvo ao observador. Para os estímulos $\mathrm{M} 1$ e $\mathrm{M} 2$ a reta de regressão seria $y=10,11-2,47 x(R=\simeq 0,53, p<0,001)$; entretanto, para o par M3 e M4 a reta de regressão seria $y=29,78 \simeq 2,74 x$ ( $R=-0,53, p<0,001)$, existindo uma diferença de 0,3 na inclinação de ambas retas, indicando que o mesmo fenômeno de compressão estaria presente nas estimativas realizadas sob ambas Diagonais. Desta maneira, o parâmetro a mostra que à medida que aumenta a distância física ao estímulo, aumenta também a subestimação da distância egocêntrica percebida. $O$ parâmetro $b$ exibe um erro constante de $\simeq 10 \mathrm{~cm}$ para a Diagonal 1, mas no caso da Diagonal 2 o valor é três vezes maior $(\simeq 30 \mathrm{~cm})$, o que poderia estar indicando que os observadores estariam considerando as diferenças espaciais entre ambas séries de estímulos e sua posição (egocentro) ao efetuar seus julgamentos.

Tomando como segmentos orientados as porções de reta determinados pelos pares de estímulos, para analisar a distância exocêntrica foi 
considerado o ângulo visual subtendido por eles nas posições de colinearidade geométrica e depois comparada com os valores médios dos ajustes efetuados pelos observadores, os quais indicariam a colinearidade perceptual. Os resultados se encontram resumidos na Tabela 4.

Tabela 4. Ângulos visuais subtendidos pelos pares de estímulos nas posições de colinearidade geométrica e segundo a média dos valores ajustados pelos observadores. Todos os valores estão indicados em graus $\left({ }^{\circ}\right)$.

\begin{tabular}{cccc}
\hline Segmento & Ângulo visual & Âng. visual Ajuste & $\Delta_{\text {ang }}$ \\
\hline F1-M1 & 41,116 & 41,379 & 0,263 \\
M2-F2 & 3,624 & 2,173 & $-1,451$ \\
F3-M3 & 67,606 & 67,092 & $-0,514$ \\
M4-F4 & 3,323 & 1,075 & $-2,248$ \\
M1-M2 & 53,097 & 54,112 & 1,015 \\
M3-M4 & 29,716 & 31,696 & 1,979 \\
\hline
\end{tabular}

Como pode ser notado, as diferenças angulares foram pequenas. No caso dos segmentos extremos (os determinados pelos estímulos fixos e os móveis), a média das diferenças seria de $\simeq 1^{\circ}$ e, quando convertidas em distâncias, as ditas diferenças mostrariam uma subconstância na percepção da distância. Apreende-se que os erros constantes são dez vezes menores que os encontrados ao avaliar as distâncias egocêntricas. Considerando os segmentos médios (os determinados pelos estímulos móveis), as médias dos erros das diferenças também são de $\simeq-1,5^{\circ}$, mas se convertidos em distâncias, estes erros indicam uma expansão na percepção do tamanho de ambos os segmentos centrais.

\section{Discussão}

Embora pareça uma tarefa simples seguir uma linha reta no espaço físico, tal como exigido pelas tarefas de apontamento exocêntrico, os resultados mostram que, ainda num ambiente naturalístico e pleno de indícios como é o campo aberto, realizar este tipo de atividade apresenta certa dificuldade, a qual parece manifestar-se nas pronunciadas diferenças individuais (Koenderink et al., 2008). Mas, coerentemente com o observado na literatura (Cuijpers et al., 2000a, 2000b, 2001, 2002; Kelly et al., 2004; Koenderink et al.,2008), as medidas de tendência central dos resultados obtidos coincidem e revelam evidências de respostas surpreendentemente acuradas no ajuste de colinearidade, sobretudo considerando a magnitude das distâncias involucradas. 


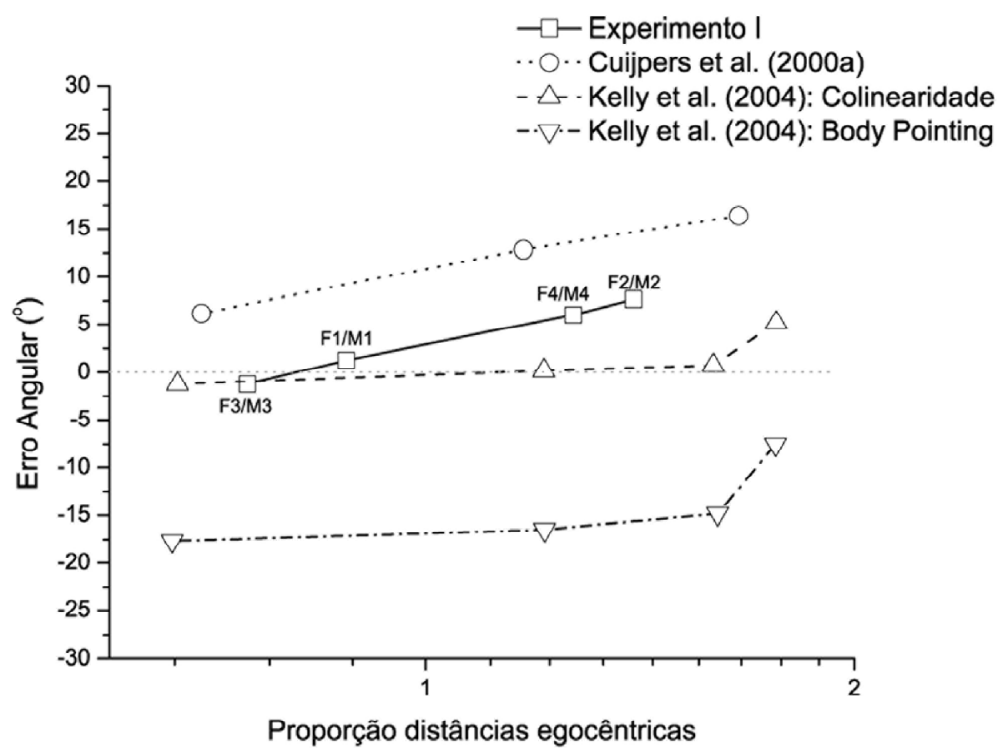

Figura 5: Média dos erros angulares (em graus) em função da proporção das distâncias egocêntricas entre os estímulos. Disposição comparativa dos dados de Cuijpers, Kappers e Koenderink (2000a) e Kelly, Loomis e Beall (2004) para esse intervalo de proporções.

Nesse sentido, evidencia-se uma forte dependência dos erros em função da distância egocêntrica aos alvos do segmento considerado. Cuijpers et al. (2000a) encontraram que tanto o tamanho como a orientação dos erros angulares são altamente dependentes da proporção entre as distâncias egocêntricas dos alvos considerados.

Kelly et al.(2004) reportaram erros similares com uma média de $7,43^{\circ}$, com a máxima para proporções entre alvos ao redor de 0,75 e a mínima para proporções ao redor de 1,3. Em nosso caso, obtivemos proporções similares e, de igual maneira, os valores maiores nos erros angulares foram reportados para as proporções de distâncias maiores. Assim, quando calculados os erros angulares da mesma forma que estes autores (considerando também o sinal na orientação dos vetores), temos para os segmentos proximais: $F 1 / M 1=0,88$ e erro angular de 1,$34 ; F 3 / M 3=0,75$ e erro angular de -1,10; e para os segmentos distais: $F 2 / M 2=1,33$ e erro angular de 7,$20 ; F 4 / M 4=1,27$ com um erro angular de 5,70. Na Figura 5 são representados estes valores e, para comparação, os dados de Kelly et al. (2004) para esse intervalo de proporções entre distâncias egocêntricas. Pode observar-se a similaridade com o padrão de erros no experimento de Cuijpers et al. (2000a) usando a tarefa de apontamento exocêntrico, onde estes autores obtiveram erros maiores provavelmente devido às condições de indícios reduzidos empregadas. Por outro lado, talvez as diferenças em relação aos resultados de Kelly et al. (2004) se devam às caracte- 
rísticas próprias implícitas em cada tarefa. No caso destes últimos autores, os observadores consideravam dois alvos fixos e projetavam mentalmente a linha de colinearidade até um terceiro ponto. Diferentemente da tarefa empregada por nós, onde os sujeitos tinham que alinhar quatro estímulos mexendo na posição de dois deles, o que implicaria um grau de dificuldade diferente por ser uma tarefa guiada visualmente.

Segundo indicam os dados, em nosso experimento a variação nas proporções das distâncias egocêntricas estaria relacionada com o ângulo na orientação dos estímulos, observando-se erros constantes específicos para cada diagonal, de acordo com Levin e Haber (1993). Estes notaram que a distância exocêntrica apresenta uma distorção que depende do ângulo entre os objetos considerados. Assim, enquanto Cuijpers et al. (2000a) encontraram uma simetria direita-esquerda na anisotropia do espaço visual, na presente investigação apreende-se que os erros são similares por diagonal e não por metade do campo visual. Ainda, estes autores asseguraram que os desvios da acurácia se reduzem quando os ângulos entre os objetos são amplos. Isto parece dever-se à falta de sensibilidade da tarefa de apontamento exocêntrico para julgar distâncias com este tipo de ângulos, obtendo dados similares com tarefa de paralelismo (Cuijpers et al., 2000b). De forma similar, encontramos uma relação inversamente proporcional entre os ângulos visuais e os erros de colinearidade cometidos. Mas é importante notar que no layoututilizado as posições dos estímulos levam à existência de uma dependência do ângulo visual entre pares de estímulos com as respectivas distâncias egocêntricas.

As pequenas variações na distância exocêntrica percebidas e a relação dos erros cometidos com as distâncias egocêntricas indicariam que o marco de referência eliciado por esta configuração de estímulos, embora possa estar baseado nos indícios exocêntricos, é escalado por um fator egocêntrico, como encontrado por Aznar-Casanova et al. (2008) e fora proposto por Foley (1980) e Foley et al.(2004). Isto também indicaria que nosso sistema visual não precisa processar a informação de distância exocêntrica para produzir estimações de orientação exocêntrica quando a informação de perspectiva está presente (caso contrário:ver AznarCasanova et al., 2008). 


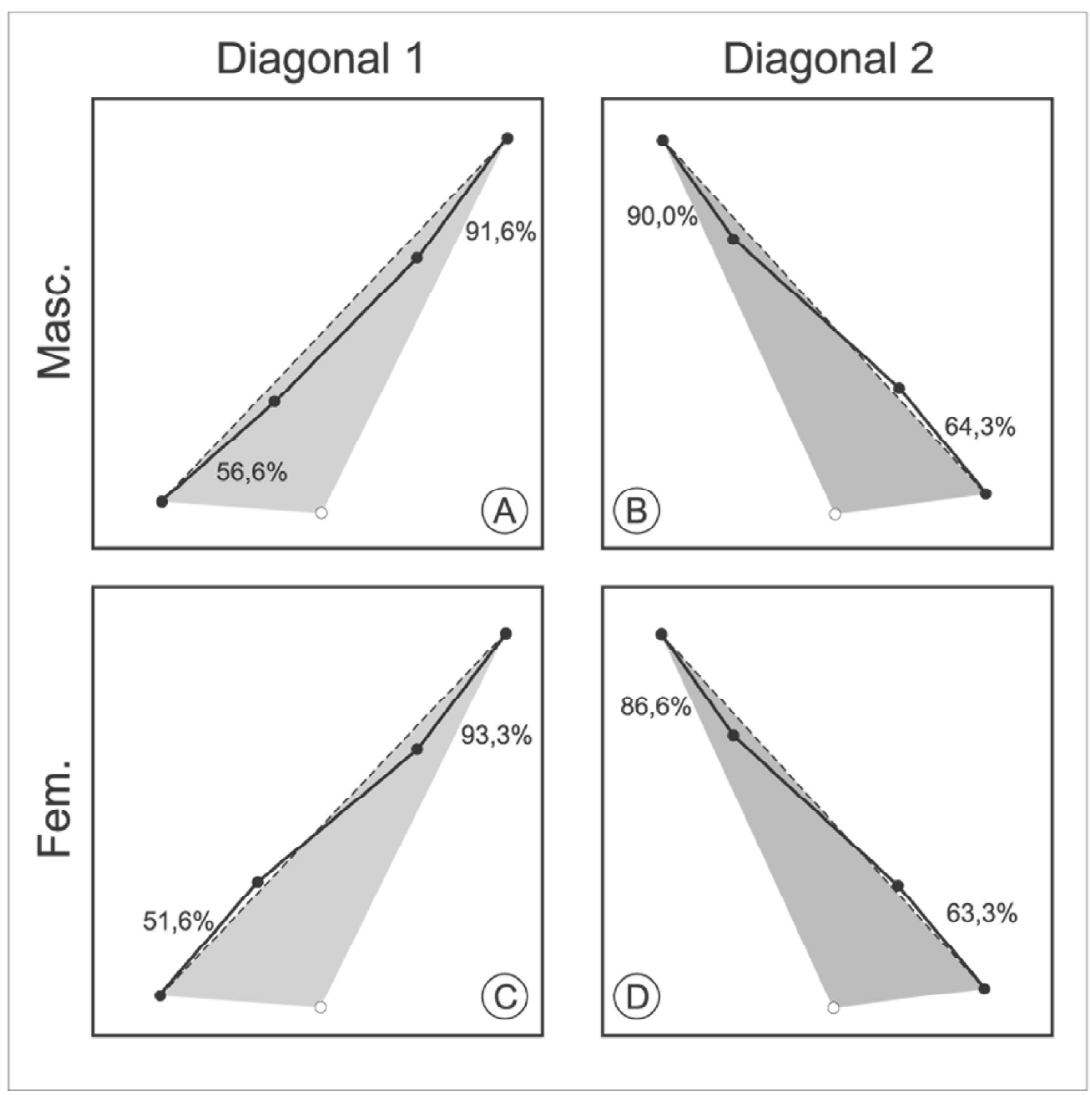

Figura 6: Orientações mais freqüentes dos estímulos em função da linha de colinearidade e do sexo do observador. Os círculos preenchidos representam os estímulos, e o círculo vazado a posição do observador.

Além das diferenças individuais entre os observadores ${ }^{7}$, assim como Koenderink et al. (2008), encontramos que, em geral, os pares de posições determinadas com os estímulos móveis falham em definir um arco simples, isto é, um arco cuja curvatura não muda de sinal. Pelo contrário, a tendência é de aparecer um ponto de inflexão entre os pontos extremos do arco apresentando um formato de "S". Estes autores acharam que as médias dos posicionamentos na maioria dos casos (91\%) estabelecem arcos com o segmento próximo ao observador apontando no sentido oposto a ele e com o segmento distante apontando para ele. Os resultados de nosso experimento mostram um padrão similar para os ângulos e distâncias empregados. Na Figura 6 se desenham, a modo de exemplo, as orientações mais frequentes. Empregam-se segmentos retos para simpliNorman, Todd, Perotti e Tittle (1996); Norman, Crabtree, Clayton e Norman (2005). 
ficar. Pode ser observado que na maioria dos casos as porcentagens indicam a característica configuração em forma de "S" (obviamente são possíveis outras combinações de orientações além das representadas). O gráfico (A) mostra o caso onde os segmentos estão orientados do mesmo lado - ou forma de "C"-, entretanto, os gráficos (B), (C) e (D) apresentam alternâncias que derivam na forma de "S".

A alta percentagem de arcos pregeodésicos encontrados (Koenderink et al., 2008) (que apareceriam na Figura 6 como linhas quebradas) mostrariam a anisotropia do espaço visual, apoiando a hipótese da não homogeneidade do mesmo. Este fato é de considerável interesse, pois levaria a descartar geometrias constantes para modelar o espaço visual, tal como aconteceria com a proposta de Luneburg (1947), ou inclusive pode ser caracterizado exclusivamente por uma geometria particular (Lukas, 2001; Norman et al., 2005). A proposta de Koenderink et al. (2008) seria de postular o espaço como 'contextual' (dependente do que estaria presente nele), ou 'momentâneo' (dependente de onde o observador esta posicionado no espaço), ou 'dependente da tarefa' (o que já tinha sido notado por Norman et al. (2005), ou talvez de combinações destas possibilidades. Foley et al. (2004) também concluíram no seu estudo que "mesmo que a percepção da posição e de extensão estejam relacionadas, não estão por uma geometria Euclidiana ou por qualquer geometria métrica".

Sem embargo, a noção do espaço visual variando em função das condições de visão e das exigências da tarefa não é paradoxal, especialmente, considerando os dados psicobiológicos que mostram a estrutura anisotrópica do sistema visual, a falta de uniformidade no campo visual (Fukusima \& Faubert, 2001; Sakaguchi, 2003) e a capacidade do sistema visual de atribuir pesos diferentes a uma mesma fonte de informação para situações diferentes de disponibilidade de indícios visuais (Cutting \& Vishton, 1995; Philbeck \& Loomis, 1997). De acordo com a perspectiva representacionista, um espaço visual isomórfico e homogêneo só poderia derivar de uma construção no percurso do tempo, incluindo nesta montagem as variações instantâneas de direções de observação devido a movimentos oculares, da cabeça, do corpo, dos objetos na cena etc. Esta construção discreta e serial necessariamente involucraria processos de memória e cognitivos (Hollingworth \& Hollingworth, 2004; Pertzov, Avidan e Zohary 2009). 


\section{Judgment of collinearity and exocentric distance perception in open field}

Abstract: The purpose was to investigate experimentally the observers' performance in an original task based on judgments of collinearity in the open field (dimensions $20 \times 30 \mathrm{~m}$ ). Thirty subjects participated in the experiment. The stimuli were vertical stakes of different heights, four fixed and four movable. The task was to adjust the two moving stimuli (via a remote control) in order to put them collinear with the other two stimuli fixed on the ends of each diagonal. An analysis of variance (ANOVA) was carry on, revealing only an significant effect for the factor 'Mobile Stimulus' $\left(F_{(3,473)=} 69,86 p<0,001\right)$ and a systematicity on the distribution of errors. The small variations in the perceived exocentric distance (with constant errors ten times lower than those found when assessing the egocentric distance) and the relationship with the errors made when estimating the egocentric distances may indicate that the frames of reference elicited by this task of colinearity, although it may be based on exocentric cues, is scaled by an egocentric factor.

Keywords: Visual perception. Space perception. Distance perception. Collinearity perception. Psychophysics.

\section{Évaluation de colinéarité et de la perception de la distance exocentrique en champ ouvert}

Résumé: Le but était d'étudier expérimentalement les performances d'observateurs pour évaluer la colinéarité en plein champ (dimensions $20 \times 30 \mathrm{~m}$ ). 30 observateurs ont participé à l'expérience. Les stimuli ont été pieux verticaux de différentes hauteurs, quatre fixes et quatre mobiles. La tâche consistait à ajuster les deux stimuli en mouvement (par l'intermédiaire d'une télécommande) afin de mettre les deux stimuli colinéaires fixées sur les extrémités de chaque diagonale. Une analyse de variance (ANOVA) des erreurs de colinéarité révélé un effet significatif seulement pour les stimulation de mobile le facteur $\left(F_{(3,473)}=69,86 p<0,001\right)$ et une cohérence dans la distribution des erreurs. Les erreurs dans la perception de la distance exocentrique sont beaucoup plus petits que ceux de la distance égocentrique.Cette relation semble indiquer que le cadre générés par cette tâche de colinéarité, même si elle peut être fondée sur la preuve exocentrique, est multipliée par un facteur égocentrique.

Mots-clés: Perception visuelle. Perception spatiale. Perception de la distance. Perception de la colinéarité. Psychophysique. 


\section{Juicio de colinearidad y percepción de distancia exocéntrica en campo abierto}

Resumen: El propósito fue investigar experimentalmente el desempeño de los observadores en una original tarea de juicio de colinearidad en campo abierto (dimensiones $20 \times 30$ m). Participaron del experimento 30 observadores. Los estímulos fueron estacas verticales de alturas variables, cuatro fijos y cuatro móviles. La tarea consistió en ajustar los dos estímulos móviles (mediante un control a distancia) para que quedaran colineares a los estímulos fijos en los extremos de cada diagonal. Se realizó un análisis de varianza (ANOVA) factorial de los errores de colinearidad, revelando solamente un efecto significativo para el factor 'Estímulo móvil' ( $F_{(3,473)=} 69,86$ $p<0,001)$ y una sistematicidad en la distribución de los errores. Las pequeñas variaciones en la distancia exocéntrica percibida (con errores constantes diez veces menores que los encontrados al evaluar las distancias egocéntricas) y la relación de los errores cometidos con las distancias egocéntricas indicarían que el marco de referencia suscitado por esta tarea de colinearidad, aunque pueda estar basado en los indicios exocéntricos, es escalado por un factor egocéntrico.

Palabras clave: Percepción visual. Percepción espacial. Percepción de distancia. Percepción de colinearidad. Psicofísica.

\section{Referências}

Aznar-Casanova, J. A., Matsushima, E. H., Da Silva, J. A., \& Ribeiro-Filho, N.P. (2008). Can exocentric direction be dissociated from its exocentric distance in virtual environments? Perception \& Psychophysics, 70(3), 541-550.

Cuijpers, R. H., Kappers, A. M., \& Koenderink, J. J. (2000a). Investigation of visual space using an exocentric pointing task. Perception \& Psychophysics, 62(8), 1556-1571.

Cuijpers, R. H., Kappers, A. M., \& Koenderink, J. J. (2000b). Large systematic deviations in visual parallelism. Perception, 29(12), 1467-1482.

Cuijpers, R. H., Kappers, A. M., \& Koenderink, J. J. (2002). Visual perception of collinearity. Perception \& Psychophysics, 64(3), 392-404.

Cutting, J.E., \& Vishton, P.M.(1995).Perceiving layout and knowing distances:The integration, relative potency, and contextual use of different information about depth.In W.Epstein \& S.J.Rogers (Eds.), Perception of space and motion (Vol.5, pp.69-117).San Diego, CA: Academic Press. 
Da Silva, J.A. (1985).Scales for perceived egocentric distance in a large open field: Comparison of three psychophysical methods. American Journal of Psychology, 98(1), 119-144.

Foley, J. M., Ribeiro-Filho, N. P., \& Da Silva, J. A. (2004). Visual perception of extent and the geometry of visual space. Vision Research, 44,147-156.

Fukusima, S. S., \& Faubert, J. (2001). Perceived length in the central visual field: evidence for visual field asymmetries. Vision Research, 41(16), 2119-2126.

Fukusima, S.S., Loomis, J.M.,\& Da Silva, J.A. (1997).Visual perception of egocentric distance as assessed by triangulation. Journal of Experimental Psychology: Human Perception and Performance, 23(1),86-100.

Gilinsky, A. S. (1951). Perceived size and distance in visual space. Psychological Review, 58(6), 460-482.

Gogel,W.C.(1978). Size, distance, and depth perception. In E.C.Carterette \& M.P. Friedman (Eds.), Hadbook of perception: Perceptual processing (Vol. 9, pp. 299-333). New York: Academic Press.

Haber, R. H. (1985). Toward a theory of the perceived spatial layout scenes. Computer Vision and Imaging Processing, 34,282-321.

Hollingworth, A., \& Hollingworth, A. (2004). Constructing visual representations of natural scenes:The roles of short- and long-term visual memory. Journal of Experimental Psychology. Human Perception and Performance, 30(3),519537.

Kelly, J.W., Loomis, J.M., \& Beall, A.C. (2004). Judgments of exocentric direction in large-scale space. Perception, 33(4), 443-454.

Koenderink, J. J., \& van Doorn, A. J. (1998). Exocentric pointing. In L. Harris \& M. Jenkin (Eds.), Vision and action (pp. 295-313). Cambridge: Cambridge University Press.

Levin, C. A., \& Haber, R. N. (1993). Visual angle as a determinant of perceived interobject distance. Perception \& Psychophysics, 54,250-259.

Loomis, J. M., Philbeck, J. W., \& Zahorik, P. (2002). Dissociation between location and shape in visual space. Journal of Experimental Psychology: Human Perception and Performance, 28, 1202-1212.

Luneburg, R. K. (1947). Mathematical analysis of binocular vision. Princeton NJ: Princeton University Press. 
Norman, J. F., Crabtree, C. E., Clayton, A.M., \& Norman, H. F.(2005). The perception of distances and spatial relationships in natural outdoor environments. Perception, 34(11), 1315-1324.

Norman, J. F., Todd, J. T., Perotti, V. J., \& Tittle, J. S. (1996). The visual perception of three-dimensional length. Journal of Experimental Psychology: Human Perception and Performance, 22,173-186.

Pertzov, Y.,Avidan, G., \& Zohary,E.(2009). Accumulation of visual information across multiple fixations. Journal of Vision, 9(10), 1-12.

Philbeck, J.W., \& Loomis, J. M. (1997). Comparison of two indicators of perceived egocentric distance under full-cue and reduced-cue conditions. Journal of Experimental Psychology:Human Perception and Performance, 23(1),72-85.

Ribeiro-Filho, N.P.(1993). Percepção de configurações espaciais de estímulos em grande campo aberto.Tese de Doutorado, Universidade de São Paulo, Ribeirão Preto, SP.

Sakaguchi,Y.(2003).Visual field anisotropy revealed by perceptual filling-in. Vision Research, 43(19), 2029-2038.

Sedgwick, H. A. (1986). Space perception. In K. R. Boff, L. Kaufman \& J.P. Thomas (Eds.), Handbook of human perception and performance:Sensory processes (Vol. 1, pp. 21.1-57). New York:Wiley.

Toye, R.C.(1986). The effect of viewing position on the perceived layout of space. Perception \& Psychophysics, 40(2), 85-92.

Wagner, M. (1985). The metric of visual space. Perception \& Psychophysics, 38, 483-495. 
Javier Enrique Santillán, Laboratório de Psicofísica e Percepção, Departamento de Psicologia e Educação, Faculdade de Filosofia, Ciências e Letras de Ribeirão Preto, Universidade de São Paulo. Endereço para correspondência: Av. Bandeirantes, 3900, CEP: 14040-901, Ribeirão Preto, SP, Brasil. Endereço eletrônico: jesantillan@gmail.com

Susi Lippi Marques, Laboratório de Investigação Psicofísica e Psicossocial-LIPP, Departamento de Psicologia, Universidade Federal de São Carlos. Endereço para correspondência: Rodovia Washington Luiz, Km 235, Monjolinho, CEP: 13565-905, São Carlos, SP, Brasil. Endereço eletrônico: susilmo@ufscar.br

José Aparecido Da Silva, Laboratório de Psicofísica e Percepção, Departamento de Psicologia e Educação, Faculdade de Filosofia, Ciências e Letras de Ribeirão Preto, Universidade de São Paulo. Endereço para correspondência: Av. Bandeirantes, 3900, CEP: 14040-901, Ribeirão Preto, SP, Brasil. Endereço eletrônico: jadsilva@ffclrp.usp.br 